\title{
炼化企业设备的本质安全可靠与监管智能化对策研究
}

\author{
王庆锋 ${ }^{1}$, 刘家赫 $^{1}$, 柳建军 ${ }^{2}$, 王学斌 $^{2}$, 李中 $^{1}$ \\ (1. 北京化工大学机电工程学院，北京 100029；2. 中国石油庆阳石化公司，甘肃庆阳 745002）
}

摘要：国内炼化企业设备事故频发，设备本质安全可靠运行面临众多挑战，其中设备可靠性设计/制造、风险管理、监测控 制智能化是工程迫切需求。为提升炼化企业设备的本质安全可靠与监管智能化水平, 本文揭示了设备本质安全可靠与监管智 能化内涵, 阐述了可靠性设计 / 制造、风险管理、监测控制智能化技术发展趋势, 提出了设备本质安全可靠与监管智能化的 对策、措施和建议。完成了某催化装置的主风机本质安全可靠与监管智能化改造案例研究, 结果表明设备本质安全可靠与监 管智能化改造对设备安全运行具有重要工程意义。

关键词: 本质安全; 可靠性设计；可靠性制造；风险管理；监管智能化

中图分类号：TH17 文献标识码：A

\section{Intrinsic Safety \& Reliability and Supervision Intelligentization of Equipment in Refinery Enterprises}

\author{
Wang Qingfeng ${ }^{1}$, Liu Jiahe ${ }^{1}$, Liu Jianjun ${ }^{2}$, Wang Xuebin ${ }^{2}$, Li Zhong ${ }^{1}$ \\ (1. College of Mechanical and Electrical Engineering, Beijing University of Chemical Technology, Beijing 100029, China; \\ 2. PetroChina Qingyang Petrochemical Company, Qingyang 745002, Gansu, China)
}

\begin{abstract}
Equipment accidents occured frequently in refinery enterprises in China, which brings great challenges on the intrinsic safety and reliability of the equipment. Equipment reliability design/manufacturing, risk management, and monitoring and control intelligentization have become urgent engineering requirements. This study reveals the connotation of intrinsic safety \& reliability and supervision intelligentization of equipment, and clarifies the development trends of reliability design/manufacturing, risk management, and monitoring and control intelligentization technologies. It also proposed corresponding countermeasures for improving the intrinsic safety and reliability and realizing supervision intelligentization of the equipment. These measures were applied to the transformation of the main fan of a catalytic unit. Results show that this type of equipment transformation is conductive to the safe operation of equipment.
\end{abstract}

Keywords: intrinsic safety; reliability design; reliability manufacturing; risk management; supervision intelligence

收稿日期 : 2018-10-26; 修回日期 : 2019-08-25

通讯作者: 王庆锋, 北京化工大学机电工程学院副研究员、硕士研究生导师, 主要研究方向为设备故障诊断与自愈化、装置可靠性与风险评估、 在役再制造; E-mail: wangqf2422@163.com

资助项目：中国工程院咨询项目 “炼化企业设备本质安全可靠与监管智能化对策研究” (2016-XZ-08)

本刊网址 : www.engineering.org.cn/ch/journal/sscae 


\section{一、前言}

2005-2016 年我国炼化企业事故频发, 引起 国家的高度关注。2005 年 11 月 13 日, 中国石油吉 林石化公司双苯厂硝基苯精馏塔发生爆炸并引发松 花江跨境水污染事件; 2011 年 9 月 8 日，上海赛科 石油化工有限责任公司超临界乙烯输送管道某流量 计旁路闸阀的阀盖与阀体突然分离, 乙烯泄漏引发 爆炸和火灾; 2013 年 11 月 22 日, 中国石油化工股 份有限公司管道储运分公司东黄输油管道原油泄漏 发生爆炸；2015 年 4 月 21 日，中国石化扬子石油 化工有限公司烯烃厂乙二醇 T-430 塔发生爆炸。大 型炼化装置事故频发，不仅经济损失巨大，还给国 家造成了严重的社会或国际影响 [1]。2016 年年底, 我国炼油能力已达到 $7.5 \times 10^{8} \mathrm{t} / \mathrm{a}$, 其中 $1 \times 10^{7} \mathrm{t} / \mathrm{a}$ 级炼厂 24 座, 合计炼油能力达到 $3.14 \times 10^{8} \mathrm{t} / \mathrm{a}$, 约 占全国炼油能力的 $42 \%$ 。大型化、高速化、自动化、 智能化的炼化装备制造成为国家战略 [2]。大型装 备创造巨大经济利益的同时，一旦发生事故，轻则 造成生产中断给企业带来巨大经济损失 [3], 重则 造成严重的安全环保事故给国家造成严重社会影 响甚至国际性影响。炼化设备本质安全与监管智 能化对于炼化企业安全可靠、清洁环保运行起到 重要的作用。

国内外对本质安全与监管智能化的理念进行了 很大程度的拓展, 在设备本质安全可靠运行及可靠 性设计 / 制造等方面开展了相关研究。许正权等 [4] 分析了国内本质安全化管理研究现状及存在问题, 提出了基于交互式安全管理理论的本质安全化管理 研究的基本思路。王钦方 [5] 根据工业发展的实际 需求，提出了包括人员、机具设备、工作环境、管 理和文化 5 个模块在内的企业本质安全化模型。吴 宗之等 [6] 将本质安全思想与 “计划 - 执行 - 检查 改进” (PDCA) 管理模式融合, 提出了具有可操作 性的基于本质安全化的 PDCA 管理模式。白晶 [7] 通过分析设备的危害因素及其控制原则以及实现设 备安全的途径, 提出加强设备的安全管理重点应 为设备的本质安全化及设备使用过程的安全管理。 Woo [8] 阐述了可靠性嵌入式设计过程及其方法发 展的必要性，建议将新参数寿命加速测试（ALT） 引入到可靠性方法中。Perng 等 [9] 在监管智能化方 面进行了研究, 提出了两种类型的智能监管控制器,
性能达到或超出线性控制限制, 优于模糊控制器和 神经控制器。Miao 等 [10] 研究了复杂多变的工业 控制系统，总结了智能工业控制综合应用的计算机、 通信、人工智能、信息和决策等技术，概述了智能 工业控制的发展趋势。Dai 等 [11] 以基于状态监测 的可靠性分析方法为主线，提出了基于条件信息的 信号选择和可靠性评估方法。

本文立足炼化设备本质安全可靠与监管智能化 研究现状, 力求揭示本质安全可靠与监管智能化的 内涵与特征; 通过梳理炼化设备本质安全可靠与监 管智能化发展趋势，明确炼化企业面临的挑战; 针 对设备本质安全可靠与监管智能化提出了对策、措 施和建议; 最后给出了国内相关工程应用案例。

\section{二、炼化设备本质安全可靠与监管智能化的 内涵与特征}

\section{（一）炼化设备本质安全可靠与监管智能化的内涵}

国外的本质安全理论研究集中在系统可靠性理 论方面, 更偏重于技术因素, 研究思路逐渐从安全 评价转向问题解决方案设计, 由此来提高系统整体 安全性 [12]。本质安全是指运用组织架构设计、技 术、管理、规范及文化等多种手段，在保障人、物 及环境可靠的前提下, 通过理顺系统在运行过程中 的基本交互关系、规范交互关系及文化交互关系， 从源头上预防系统在运行中出现的反效交互、无效 交互及 II 型有效交互所引起的系统不和谐, 进而保 证实现系统安全、设备可靠、管理全面及安全文化 深入人心，最终达到对事故的长效预防 [4]。本质 安全贯穿于设计、制造、运行、维修等设备全生命 周期的各个阶段：设计阶段采用本质安全技术，留 足安全系数，采用安全装置（固定、联锁、控制、 跳闸等形式）实现机械化和自动化; 制造阶段落实 加工、装配、测试等标准, 确保零部件的在役可靠 性; 运行阶段采用的控制系统设计应考虑各种作业 的操作模式，具有故障显示、自动监控、人工干预 等功能; 维修阶段采用物联网监测、故障诊断、基 于风险的维修管理等技术实现精准维修, 保证设备 始终处于可靠和可用状态。

设备本质安全可靠与监管智能化的内涵可定义 为: 以设备的可靠性设计和制造为根本，以风险管 理手段为核心，以监测控制智能化和信息化技术为 
支撑，实现设备实时状态感知、状态辨识与预测、 外部环境扰动或自身故障自适应调节控制和自主式 维修保障决策, 确保设备运行可靠安全和人、机、 环境和谐。可靠性设计 / 制造、风险管理、监测控 制智能化和信息化是确保设备本质安全可靠的 3 个 关键要素。

\section{（二）炼化设备本质安全可靠与监管智能化特征}

1. 以可靠性设计/制造为根本

装备在规定的时间内完成规定功能的能力即为 可靠性。可靠性设计包含质量、成本、可靠度三方 面, 优化过程把产品的总体可靠度作为性能约束, 会产生与合理安全性相协调的平衡设计 [13]。可靠 性设计/制造是指在设备设计和制造方面从设备的 本质安全出发, 为消除设备的潜在缺陷和薄弱环节、 防止故障发生，以确保满足规定的固有可靠性要求 所采取的技术活动。设备运行可靠性约 $90 \%$ 是由 可靠性设计/制造决定的。可靠性设计/制造能保 证设备的本质安全, 杜绝或减少伤亡事故, 减少设 备故障, 从而提高设备利用率, 实现安全生产; 设 备的设计选型也要选择有可靠性设计/制造保证的 产品。

2. 以风险管理为全生命周期管理的核心

风险管理是指利用风险辨识、风险分析、风险 定量计算、可靠性管理等手段保证设备正常、安全 的运行状态 [14]。针对动静电仪设备开展以可靠性 为中心的维修 (RCM)、基于风险的检验（RBI）、 安全完整性等级（SIL）等风险评估, 找出影响设 备可靠运行的关键因素, 采用故障根除措施, 制定 降险措施或一次性变更任务, 提高设备本质安全可 靠性。风险管理技术贯彻到设备设计、建造、安装、 运行、维护、报废等设备全生命周期的各个阶段。

3. 监测控制以智能化信息化为技术支撑

“监测与预警、诊断与预测” 成为设备监测诊 断的发展方向。利用工业互联、移动互联、状态监 测与诊断、信息化云平台技术，通过数据把人和机 器联系起来, 实现设备以可靠性为中心的维修, 提 高人的工作效率、确保设备运行安全。

4. 以全面感知、状态辨识与预测、自主维修决 策为实现途径

基于炼化设备 RCM 评估，针对高风险故障模 式开展故障可探测性分析, 确定故障特征敏感参数,
给机器安装各类传感器使其具备故障感知能力; 给 机器配置信号处理分析的 “大脑”, 利用设备健康 度评价模型、能效评价模型、可靠性评价及其相应 的评价绩效标准, 使机器具备状态辨识与预测能力; 让机器连接网络, 使状态辨识与预测信息通过网络 实现故障预警; 借助于 RCM 维修决策知识库, 自 主决策维修内容。

5. 以外部环境扰动或自身故障的自适应调节控 制为技术保障

在系统论的指导下，打破传统的专业壁垒，借 鉴和移植现代医学 “自主调理” 治疗原理, 集成状 态监测诊断、人工智能、主动和自适应控制等技术, 研究以事故预防和自愈为目标的过程自主调控, 使 炼化企业设备具备故障自愈功能, 从而大幅度减少 故障和事故、减少停机生产损失。

6. 故障根除任务是有效途径

设备维修只能保持而不能提高设备固有的可靠 性, 自主式维修保障决策就是决定最佳的维修时机 和维修内容, 做到避免 “维修不足” 或 “维修过剩”。 故障根除任务是自主式维修保障决策的重要内容 之一, 针对高风险故障模式, 开展故障根源分析, 制定故障根除措施，是提高设备固有可靠性的有 效途径 [15]。

\section{三、设备本质安全可靠与监管智能化发展趋势}

\section{（一）设备设计与制造向智能化方向发展}

1. 数字化设计与仿真技术是重要手段

在数字化集成环境下, 开展可靠性与性能一体 化的设计与仿真, 针对机械、机电等复杂产品进行 结构 / 机构、设备、系统级产品复杂环境下多场耦 合、多机理相关的可靠性量化分析、寿命预测、方 案优化等研究, 具体包括多学科工具集成、试验设 计、响应面耦合、可靠性分析、系统可靠性分析、 可靠性优化、耐久性分析 7 个功能模块。

\section{2. 机械装备智能化是发展趋势}

当今社会大量地采用智能技术，包括各种智能 生产控制系统、智能物流系统、智能制造系统等。 过程装备的自我感知、自动辨识、自动预测、外部 扰动自适应调整、故障自愈化等特性都带有典型的 智能化特征。流体机械的防喘振控制、无级气量调 节控制、联锁保护、过载保护等自控设施是智能化 
控制的基础。

\section{3. 智能制造是实现途径}

智能制造是指面向产品全生命周期, 实现泛在 感知条件下的信息化制造。在现代传感技术、网络 技术、自动化技术、拟人化智能技术等先进技术的 基础上, 通过智能化的感知、人机交互、决策和执 行技术，实现设计过程、制造过程和制造装备智能 化, 是信息技术、智能技术与装备制造技术的深度 融合与集成。充分利用网络化制造、数字化制造 的基础, 融入人工智能和机器人技术, 形成人、机、 物的交互与深度融合, 使设计、工艺、试验仿真、 生产过程、保障及管理等各阶段的智能化成为现 实 $[16,17]$ 。

\section{（二）风险管理技术将成为设备完整性管理的重要 支撑}

1. 设备完整性管理是设备管理发展趋势

设备管理模式从故障后维修 $(\mathrm{BM})$ 、计划维修 ( TBM)、状态维修 $(\mathrm{CBM}$ )、基于风险的维修 (RBM) 发展到资产完整性管理（AIM）。完整性管理贯穿 设备设计、制造、安装、运行、维修、报废等全生 命周期的各个阶段, 是炼化企业设备管理模式的主 流发展趋势。

\section{2. 风险管理是完整性管理的核心}

设计阶段应用量化风险评价 (QRA)、危害 与可操作性分析 (HAZOP)、可靠性/可用性分析 $(\mathrm{RAM}) 、 \mathrm{SIL}$ 等风险评估工具, 识别设计阶段存在 的风险因素并采用一次性变更措施, 确保设备设计 本质安全可靠。

运行、维修阶段利用设备失效分析 (FTA)、 根本原因分析 (RCA) 方法识别造成设备缺陷的潜 在因素; 静设备应用 RBI 技术进行风险分析并制定 检验计划; 动设备应用 RCM、RAM 技术进行可靠 性分析, 制定检验维修策略; 仪表电气应用 SIL 技 术进行安全完整性等级分析, 制定相应的管理策略; 操作方面采用完整性操作窗口（IOW）设定设备失 效的操作边界, 预防与干预设备状态劣化造成的事 故发生, 确保设备安全运行。

3. 风险管理促使传统的设备管理模式从制度层 面转向技术层面

传统的设备管理依靠制度规范, 明确了人要怎
么管设备。风险管理从技术层面出发, 提出设备需 要怎么管理才能确保设备安全可靠运行的同时降低 维修资源耗费和提高装置的运转周期。

\section{（三）设备监测将向网络化、智能化方向发展}

\section{1. 监测预警智能化}

常规的监测报警容易出现假报警、虚报警和反 复穿越多次报警。监测预警智能化的发展趋势表现 为取代门槛阈值报警方式, 根据设备运行工况、服 役年限等因素设置智能报警线; 报警不但分级, 而 且给出报警的位置、报警的危险级别和解除、延缓 报警的策略方法。

\section{2. 监测诊断智能化}

以多参数、大容量替代单参数监测, 平稳运行 监测发展到非平稳运行监测, 信息集成、融合、分 解、提纯等技术 (取代单参数的阈值比较) 是监测 诊断智能化发展的基础。监测诊断智能化的发展趋 势是实现设备自动故障诊断、自动故障预测和剩余 寿命预测。

\section{3. 监测诊断网络化}

实时在线监测替代定期监测和巡回监测, 分布 式、网络化监测代替集中监测, 这是监测诊断的发 展趋势。以物联网、云计算、大数据等为代表的新 一代信息与通信技术创新活跃, 发展迅猛, 正在全 球范围内掀起新一轮科技革命和产业变革, 成为推 动经济社会发展的重要力量。物联网可以将所有行 使独立功能的普通物体与互联网相连, 进行信息交 换和通信, 以实现智能化的识别、定位、跟踪、监 控和管理。从全球来看, 物联网大数据处理和公共 平台服务方兴未艾, 物联网相关的终端制造和应用 服务仍在成长培育期。

不依靠机理研究而直接从大数据出发识别设备 运行状态, 这也是炼化设备智能监管的一个趋势。 借助物联网、云计算、大数据, 充分利用炼化企业 大数据进行数据挖掘, 通过统计、在线分析、机器 学习、模式识别等方法开展数据的分类与估计、规 则关联、聚类、可视化描述等应用, 从而实现设备 故障识别、早期预警与诊断、设备健康状态在线评 估等功能。一些互联网公司开始利用人工智能技术 支持炼化企业探索智能生产, 综合采用多种网络技 术保障设备之间、设备与制造云数据中心之间的数 
据交换, 为智能工厂提供监测智能化的数据基础。

基于工业大数据和网络的制造服务, 以云计算、 数据融合处理与分析、远程监控与诊断等技术为支 撑, 建立网络远程状态监控与诊断和后勤保障系统, 支撑运营模式变革, 扩展维护、租赁和数据分析管 理等服务, 由此奠定设备本质安全可靠与监管智能 化的技术基础。

\section{四、我国炼化设备本质安全可靠与监管智能 化宏观研究}

\section{（一）我国炼化设备本质安全可靠与监管智能化存 在的问题}

设备可靠性设计/制造、风险管理和智能化监 控方面的法律、法规或规章制度不健全; 设备重特 大事故的调查往往以追责为目的, 事故往往无法追 根溯源。

设备可靠性设计/制造、风险管理和智能化监 控标准评价体系缺乏或不健全; 风险管理、监管智 能化从业人员资格未有认证制度，从业人员技术水 平有待整体性提升。

设备采购低价中标制度不能鼓励提高设计 / 制 造质量, 也难以推动设备本质安全可靠所需的安全 设施建设。

设备设计/制造监测控制智能化程度不高, 技 术水平相对落后；设计院、制造厂的产品设计相对 保守，在 “源头” 就缺乏设备故障可探测性、自适 应调控性和自愈化等功能设计。

在设备运行维护管理阶段, 一方面管理者由于 知识所限, 认为监测控制智能化设施功能 “画蛇添 足” 而不重视; 另一方面设备监测控制智能化程度 达不到用户预期功能。两种现象同时存在。

设备管理模式的传统想法根深蒂固, RBI、 RCM、SIL、HAZOP 等先进成熟的管理模式难以落 地生根。

设备决策管理靠经验、靠领导，缺乏利用设备 运行数据、监测诊断数据、检修维护数据等进行科 学决策的意识和能力。

设备本质安全可靠智能化程度低, 造成设备运 行不敢卡边操作、以牺牲运行效率换取安全的现状 普遍存在。
（二）我国炼化设备本质安全可靠与监管智能化对 策与建议

在国家层面着手建立炼化设备设计 / 制造本质 安全可靠相关的法律、法规或规章制度; 设备重大 事故根源为设计 / 制造缺陷的, 要对相关单位追责。

制定炼化设备设计/制造、风险管理、监管智 能化方面的行业标准或规范并建立定期评审、更新 机制; 以最佳可行技术为依据，提高企业在标准制 定过程中的参与程度; 对从业人员实行资格认证制 度，提高风险评估、监测诊断结论的权威性。

从设备本质安全可靠与监管智能化的内在需求 出发, 完善设备及其零部件采购管理制度; 设备运 行效率、无故障运行周期、故障平均修复时间、年 维修费用、事故安全影响和环境影响等作为重要评 价指标。

在设备设计/制造方面，开展故障可检测性设 计和工业互联设计，提升装备的故障感知能力、状 态辨识与预测能力和工业互联能力; 通过数字化、 自动化、模型化、智能化、信息化等措施提升装备 本质安全设计水平; 实现炼化企业全流程安全生产 管控一体化, 形成装备运行状态辨识与预测、自适 应规划控制与动态补偿修复、智能维修决策等能力 (见图 1)。

建立健全设备完整性管理制度体系, 实现设备 设计、制造、安装、运行、维修、报废等全生命周 期的管理。

在设备全生命周期的各个阶段，贯彻应用 RBI、RCM、SIL 等风险评估工具，成为国家或行 业性的正式要求; 炼化企业要制定与之相适应的管 理规章制度并监督执行。

利用大数据、云计算和人工智能等基础技术, 突破设备故障自动诊断、自动预测、智能预警等 核心关键技术, 构建设备完整性管理智能运维决 策信息平台，实现数据驱动的设备智能运维决策 (见图 2)。

掌握炼化设备健康与能效监控关键技术, 建立 压缩机机组智能调控系统, 利用专家系统对炼化设 备进行集中控制, 采用智能化算法优化负载分配, 使各压缩机机组运行在最优工况, 从而实现运行效 益的最大化。采用大机组防喘振优化控制和故障自 愈调控技术，在提升机组运行效率的同时，确保设 备卡边运行的安全可靠。 


\begin{tabular}{|c|c|c|c|c|c|c|c|c|c|c|c|c|c|c|}
\hline 智能办公 & \multicolumn{5}{|c|}{ 管理者驾驶舱 } & \multicolumn{3}{|c|}{ 桌面终端屏幕 } & & & \multicolumn{3}{|c|}{ 移动终端屏幕 } & 可视化 \\
\hline 智能决策 & \multicolumn{2}{|c|}{ 远程运维 } & & \multicolumn{2}{|c|}{ 监测诊断 } & & \multicolumn{2}{|c|}{ 维修决策 } & \multicolumn{3}{|c|}{ 任务优化 } & \multicolumn{2}{|c|}{$\cdots \cdots$} & 知识化 \\
\hline \multirow{3}{*}{ 智能评估 } & \multicolumn{2}{|c|}{$\begin{array}{c}\text { 动设备 } \\
\text { 健康评估 }\end{array}$} & \multicolumn{2}{|c|}{$\begin{array}{c}\text { 静设备 } \\
\text { 健康评估 }\end{array}$} & \multicolumn{2}{|c|}{$\begin{array}{l}\text { 电力设备 } \\
\text { 健康评估 }\end{array}$} & \multicolumn{2}{|c|}{$\begin{array}{l}\text { 自控设备 } \\
\text { 健康评估 }\end{array}$} & \multicolumn{3}{|c|}{$\begin{array}{c}\text { 动设备 } \\
\text { 能效评估 }\end{array}$} & \multicolumn{2}{|c|}{$\begin{array}{c}\text { 动态 } \\
\text { 风险评估 }\end{array}$} & \multirow{3}{*}{ 模型化 } \\
\hline & \begin{tabular}{|l|} 
智能 \\
预警
\end{tabular} & $\begin{array}{l}\text { 智能 } \\
\text { 预测 }\end{array}$ & 实时 & $\begin{array}{l}\text { 寿命 } \\
\text { 预测 }\end{array}$ & $\begin{array}{l}\text { 超限 } \\
\text { 预警 }\end{array}$ & $\begin{array}{l}\text { 故障 } \\
\text { 辩识 }\end{array}$ & $\begin{array}{l}\text { 超限 } \\
\text { 预警 }\end{array}$ & \begin{tabular}{|l|} 
故障 \\
辨识
\end{tabular} & & 角能 & \begin{tabular}{|l|} 
效率 \\
分析
\end{tabular} & $\begin{array}{l}\mathrm{RCM} \text { 在 } \\
\text { 线评估 }\end{array}$ & $\begin{array}{l}\text { RBI在 } \\
\text { 线评估 }\end{array}$ & \\
\hline & 智能 & & $\begin{array}{l}\text { 实时 } \\
\text { 诊断 }\end{array}$ & & $\begin{array}{l}\text { 功能 } \\
\text { 代偿 }\end{array}$ & & $\begin{array}{l}\text { 功能 } \\
\text { 代偿 }\end{array}$ & & \multicolumn{3}{|c|}{\begin{tabular}{c|c|c} 
工艺设备 & $\ldots$ \\
匹配分析 & $\ldots$
\end{tabular}} & \begin{tabular}{|l|} 
SIL在 \\
线评估
\end{tabular} & & \\
\hline 系统集成 & \multicolumn{2}{|c|}{ 数据云（新建） } & \multicolumn{4}{|c|}{ 工业大数据中心 (新建) } & \multicolumn{2}{|c|}{ MES } & \multicolumn{2}{|c|}{ ERP-PM } & \multicolumn{2}{|c|}{ EAM } & 其他........ & 集成化 \\
\hline 工业互联 & $4 \mathrm{G} / 5$ & 无线网络 & & 企业生 & 产网络 & & 企业工控网 & & & 业办公 & 网络 & 设备监 & 则网 & 数据管道 \\
\hline \multirow{3}{*}{ 状态监测 } & \multicolumn{2}{|c|}{$\begin{array}{c}\text { 动设备 } \\
\text { 状态监测 }\end{array}$} & & \multicolumn{2}{|c|}{$\begin{array}{c}\text { 静设备 } \\
\text { 状态监测 }\end{array}$} & & \multicolumn{2}{|c|}{$\begin{array}{l}\text { 电力设备 } \\
\text { 状态监测 }\end{array}$} & & & \multicolumn{3}{|c|}{ 自控设备状态监测 } & \\
\hline & $\begin{array}{l}\text { 振动 } \\
\text { 监测 }\end{array}$ & $\begin{array}{l}\text { 油液 } \\
\text { 监测 }\end{array}$ & & $\begin{array}{l}\text { 腐蚀 } \\
\text { 监测 }\end{array}$ & $\begin{array}{l}\text { 无损 } \\
\text { 检测 }\end{array}$ & & $\begin{array}{l}\text { 电力 } \\
\text { 监测 }\end{array}$ & $\begin{array}{l}\text { UPS } \\
\text { 监测 }\end{array}$ & & & $\begin{array}{l}\text { 智能 } \\
\text { 变送器 }\end{array}$ & $\begin{array}{c}\text { 智能 } \\
\text { 执行器 }\end{array}$ & $\begin{array}{l}\text { 智能 } \\
\text { 特阀 }\end{array}$ & 自动化 \\
\hline & $\begin{array}{l}\text { 应力 } \\
\text { 监测 }\end{array}$ & & & $\begin{array}{l}\text { 腐蚀 } \\
\text { 防护 }\end{array}$ & & & $\begin{array}{l}\text { 温度 } \\
\text { 监测 }\end{array}$ & & & & $\begin{array}{c}\text { 智能 } \mathrm{I} / \mathrm{O} \\
\text { 卡件 }\end{array}$ & $\begin{array}{l}\text { 智能分 } \\
\text { 析仪表 }\end{array}$ & & \\
\hline 全面感知 & 位移 & 速度 & 加速 & 温度 & 压力 & 流量 & 液位 & 当压 & 电流 & 应变 & , & $\begin{array}{l}\text { 瞬时㾊 } \\
\text { 蚀速率 }\end{array}$ & 氢通量 & 数字化 \\
\hline
\end{tabular}

图 1 动静电仪设备本质安全可靠与监管智能化设计框架图

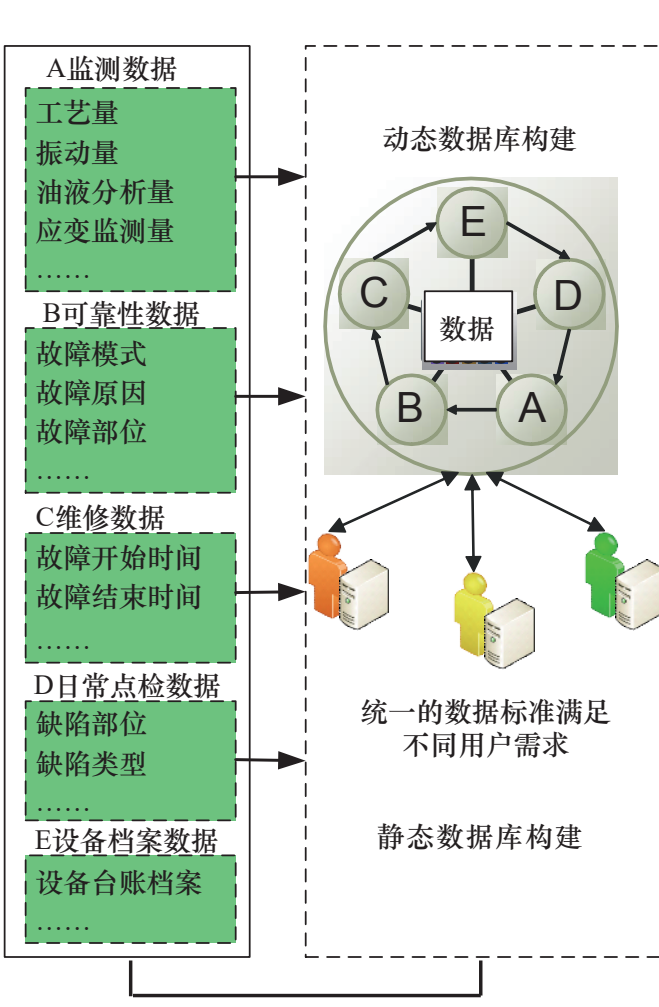

实时状态感知

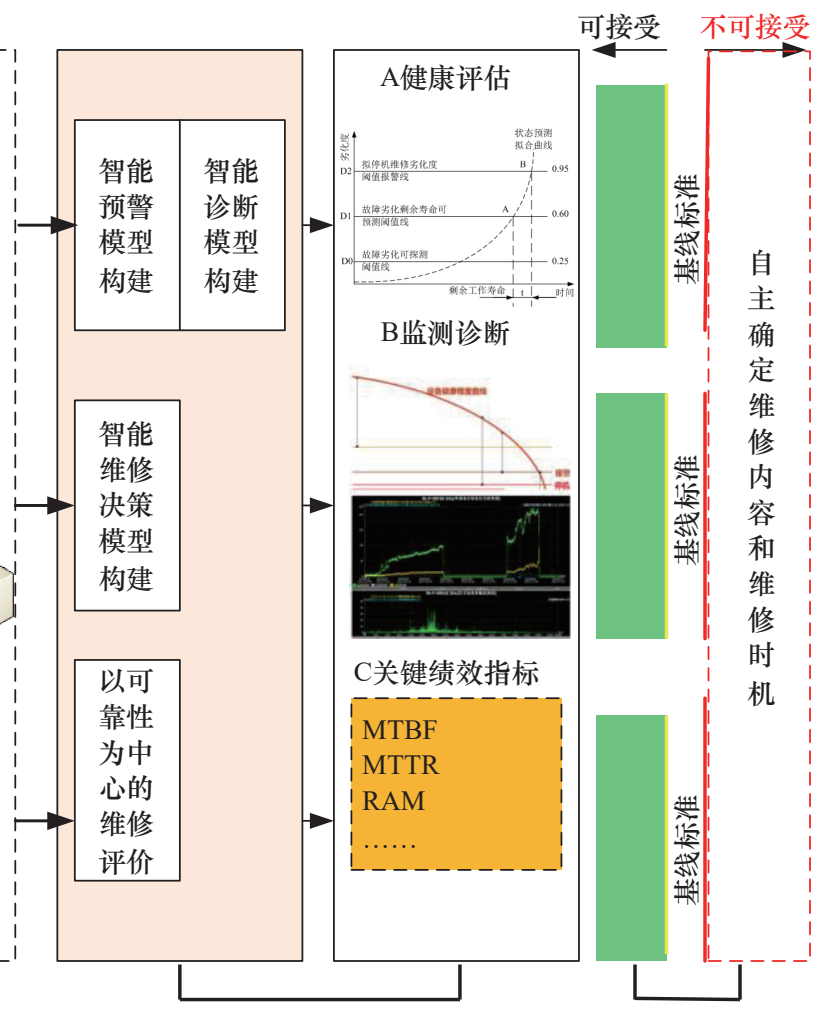

状态辨识与预测

智能维修决策 


\section{五、炼化设备本质安全可靠与监管智能化技 术的工程应用案例}

采用炼化设备本质安全可靠与监管智能化技术 进行了工程案例实施 [18]。中国石油化工集团有限 公司某子公司催化装置的主风机在使用的 8 年时间 内曾数次出现卡件、系统报警和联锁误动作等故障, 2011 年 2-4 月期间主风机出现两次静叶漂移关闭 到 $22^{\circ}$ 位置致使系统停车。据不完全统计, 国内相 关单位平均每年因主风机静叶可调机构锁位或跑位 故障造成系统停产为 3 5 次, 平均每次系统停产造 成的损失约为 3024 万元。为减少系统停产所造成 的损失, 保障设备安全运行, 针对该主风机系统开 展本质安全可靠智能化监管改造。

\section{（一）本质安全可靠智能化监管对象确定}

可靠性数据和维修数据统计分析表明, 主风机 常规电液控制系统的故障概率 (PFD) 达到了 2.5207, 而同期先进水平控制系统 PFD 仅为 0.1 。主风机本 质安全可靠智能化监管对象据此确定为主风机静叶 可调执行机构常规电液控制系统。

\section{（二）可靠性关键影响因素及个性化再设计}

影响主风机运行可靠性的主要因素在于, 主 风机静叶可调执行机构的常规电液控制系统的特阀 存在 “堵、卡、漂” 现象, 同时智能化程度低。基 于可靠性设计和故障仿生自愈原理, 研制具有自诊 断及自愈化为特征的智能化电液控制系统, 提出一 种基于功能代偿的多靶点电液控制系统故障自愈调 控方法。基于集散控制系统 / 安全仪表系统（DCS/ SIS）平台开发了主风机静叶可调执行机构电液控 制故障自愈调控系统, 使静叶可调执行结构的故障 可探测性和自适应调控性得到了根本性提高。

\section{（三）本质安全可靠智能化监管改造效果}

在技术性方面, 电液控制性实现了状态感知、 故障可探测、静叶可调执行机构阀位锁位或跑位故 障自愈调控。

在经济性方面, 具有自愈调控功能的智能化 电液控制系统研制成本为 120 万元，每年按照减少 1 次系统停车计算预期收益为 3024 万元, 投入 / 产 出比仅为 0.03968 。
在服役性方面, 智能电液控制性 PFD 仅为 0.005864 ; 系统实现了在线不停产维修, 平均修 复时间（MTTR）不大于 $2 \mathrm{~h}$; 设计服役时限可达 $3 \times 10^{5} \mathrm{~h}$ 。

在资源性方面, 智能电液控制运行功耗为 $0.47 \mathrm{~kW}$, 优于国内最好水平 $(12 \mathrm{~kW})$, 单位原油 加工电耗、蒸汽消耗平均降低 $0.5 \%$ 。

在环境性方面, 主风机运行可靠性显著提高, 系统停产造成的气体、液体排放总量降低 1 个数量 级以上。

\section{六、结论}

本文揭示了设备本质安全可靠与监管智能化 的内涵与特征。可靠性设计/制造、风险管理、监 测控制智能化和信息化是确保设备本质安全可靠的 3 个关键要素。梳理了炼化设备本质安全可靠与监 管智能化发展趋势: 设备设计与制造向智能化方向 发展, 风险管理技术将成为设备完整性管理的重要 支撑，设备监测将向网络化、智能化方向发展。明 确了我国炼化设备本质安全可靠与监管智能化实现 面临的挑战, 提出了设备本质安全可靠与监管智能 化的对策、措施和建议。

着眼未来，基于信息物理系统（CPS）实现设 备全面感知、早期故障预警、运行状态实时评估和 智能维修决策, 这是设备本质安全可靠与智能化监 管技术的发展趋势。

\section{参考文献}

[1] 吕运容, 陈学东, 高金吉, 等. 我国大型工艺压缩机故障情况调 研及失效预防对策 [J]. 流体机械, 2013, 41(1): 14-20.

Lv Y R, Chen X D, Gao J J, et al. Survey of failure of large process compressor in chinese and failure prevention countermeasure $[\mathrm{J}]$. Fluid Machinery, 2013, 41(1): 14-20.

[2] 国家制造强国建设战略委员会. 中国制造2025蓝皮书（2016） [M]. 北京: 电子工业出版社, 2016.

National Manufacturing Powerhouse Construction Strategy Committee. Made in China 2025 blue book (2016) [M]. Beijing: Publishing House of Electronics Industry, 2016.

[3] 高金吉, 杨国安. 流程工业装备绿色化、智能化与在役再制造 [J]. 中国工程科学, 2015, 17(7): 54-62.

Gao J J, Yang G A. Greenization, intelligentization and remanufacture in service of equipment in process industry [J]. Strategic Study of CAE, 2015, 17(7): 54-62.

[4] 许正权, 宋学锋, 李敏莉. 本质安全化管理思想及实证研究框架 [J]. 中国安全科学学报, 2006 (12): 79-85. 
Xu Z Q, Song X F, Li M L. Thoughts and empirical research frame on the inherent safety management theory $[\mathrm{J}]$. China Safety Science Journal, 2006 (12): 79-85.

[5] 王钦方. 企业本质安全化模型研究 [J]. 中国安全科学学报, 2005 (12): 33-36,138.

Wang Q F. Study on the model of essential safety in enterprise [J]. China Safety Science Journal, 2005 (12): 33-36,138.

[6] 吴宗之, 任彦斌, 牛和平, 等. 基于本质安全理论的安全管理体 系研究 [J]. 中国安全科学学报, 2007 (7): 54-58.

Wu Z Z, Ren Y B, Niu H P, et al. Study on safety management system based on inhenrent safety theory [J]. China Safety Science Journal, 2007 (7): 54-58.

[7] 白晶. 设备安全在本质安全管理中的重要性 [J]. 中国新技术新 产品, 2010 (11): 152.

Bai J. The importance of equipment safety in intrinsic safety management [J]. China New Technologies and Products, 2010 (11): 152.

[8] Woo S. Reliability design of mechanical systems: A guide for mechanical and civil engineers $[M]$. Singapore: Springer Nature Singapore Pte Ltd, 2017.

[9] Perng M H, Chang H H. Intelligent supervision of servo control [J]. IEE Proceedings D-Control Theory and Applications, 2006, 140(6): 405-412.

[10] Miao L, Lei C, Li X, et al. Research on the development of intelligent industrial control [C]. Hong Kong: International Conference on Intelligent and Interactive Systems and Applications, 2017.

[11] Dai W, Sun J, Chi Y, et al. Review of machining equipment reliability analysis methods based on condition monitoring technology [J]. Applied Sciences, 2019, 9(14): 2786.

[12] 居滋培. 可靠性工程 [M]. 北京: 原子能出版社, 2000. Ju Z P. Reliability engineering [M]. Beijing: Atomic Energy Press, 2000.

[13] 张义民. 机械可靠性设计的内涵与递进 [J]. 机械工程学报,
2010, 46(14): 167-188.

Zhang Y M. Connotation and development of mechanical realiability-based design [J]. Journal of Mechanical Engineering, 2010, 46(14): 167-188.

[14] 王庆锋. 基于风险和状态的智能维修决策优化系统及应用研究 [D]. 北京: 北京化工大学(博士学位论文), 2011.

Wang Q F. Risk and based intelligent maintenance decisionmaking optimization system and application research [D]. Beijing: Beijing University of Chemical Technology (Doctoral dissertation), 2011.

[15] 王庆锋, 高金吉. 过程工业动态的以可靠性为中心的维修研究 及应用 [J]. 机械工程学报, 2012, 48(8): 135-143.

Wang Q F, Gao J J. Research and application of dynamic reliability centered maintenance for process industry [J]. Journal of Mechanical Engineering, 2012, 48(8): 135-143.

[16] 张俊, 卢秉恒. 面向高端装备制造业的高端制造装备需求趋势 分析 [J]. 中国工程科学, 2017, 19(3): 136-141.

Zhang J, Lu B H. Requirement analysis of high-end manufacturing equipment with a focus on the high-end equipent manafactuing industry [J]. Strategic Study of CAE, 2017, 19(3): 136-141.

[17] 谭建荣, 刘达新, 刘振宇, 等. 从数字制造到智能制造的关键技 术途径研究 [J]. 中国工程科学, 2017, 19(3): 39-44.

Tan J R, Liu D X, Liu Z Y, et al. Research on key technical approaches for the transition from digital manufacturing to intelligent manufaturing [J]. Strategic Study of CAE, 2017, 19(3): 39-44.

[18] 王庆锋, 高金吉, 袁庆斌, 等. 主风机静叶可调执行机构自愈 化智能电液控制系统研究与应用 [J]. 机械工程学报, 2016, 52 (20): 185-192.

Wang Q F, Gao J J, Yuan Q B, et al. Research and application of self-recovery smart electro-hydraulic control system on axialblower static blade adjustable actuator [J]. Journal of Mechanical Engineering, 2016, 52(20): 185-192. 